\title{
Self-Assembled Hollow Nanocapsule from Amphiphatic Carboxymethyl-hexanoyl Chitosan as Drug Carrier
}

\author{
Kun-Ho Liu, ${ }^{\dagger}$ San-Yuan Chen, ${ }^{\dagger}$ Dean-Mo Liu, ${ }^{*},{ }^{\dagger}$ and Tse-Ying Liu ${ }^{*}$ \\ Department of Materials Science and Engineering, National Chiao Tung University, \\ 1001 Ta-hsueh Rd. Hsinchu, Taiwan, Republic of China, and Institute of Biomedical Engineering, \\ National Yang-Ming University, No. 155, Sec. 2, Linong St. Taipei, 112 Taiwan, Republic of China
}

Received January 31, 2008; Revised Manuscript Received June 23, 2008

\begin{abstract}
A new type of amphiphilic chitosan, which was synthesized through the use of both hydrophilic carboxymethyl and hydrophobic hexanoyl substitutions, was employed to self-assemble into a hollow nanocapsule in an aqueous environment. Critical aggregation concentration (cac) and zeta potential were experimentally identified for the amphiphilic chitosan (CHC). Both experimental data suggested that the self-assembly behavior of the $\mathrm{CHC}$ is fundamentally determined as an interplay between the hydrophobic interaction and the variation of the zeta potential upon hexanoyl substitution, which further influenced the nanostructural evolution of the nanocapsules. Higher hexanoyl substitution promoted larger nanocapsules, ca. $200 \mathrm{~nm}$ in diameter, while a reduced zeta potential was correspondingly detected, and vice versa, forming smaller nanocapsules, ca. $20 \mathrm{~nm}$ in diameter. The selfassemble mechanism, together with the corresponding nanostructural stability, of this unique CHC nanocapsule was also proposed in terms of intermolecular interaction and thermodynamic reason. By taking the advantage of the self-assemble (or self-aggregation) capability, the $\mathrm{CHC}$ was employed for drug encapsulation, i.e., doxorubicin, an anticancer molecule; we found in this preliminary evaluation that it reached an efficiency of $46.8 \%$, and a corresponding drug release from the nanocapsules for a time period exceeded 7 days can be achieved in vitro.
\end{abstract}

\section{Introduction}

Nanotechnology has received the greatest attention because of its potential that can literally revolutionize each field in which it is being exploited. The importance of nanotechnology in drug delivery is in the concept and ability to manipulate molecules and supramolecular structures for producing devices with programmed functions. In biotechnology and medicine, by controlling the composition, structure, and function of the nanosized polymer materials, such as nanoparticles, ${ }^{1}$ micelles, ${ }^{2}$ and nanocapsules, ${ }^{3}$ they can serve as effective vehicles for drug delivery, drug controlled release, and gene therapy. Compared to nanoparticle (monolithic-type device), hollow nanocapsules (reservoir-type device) have been attracting increasing interest because of their potential ability to encapsulate large quantities of therapeutic and diagnostic agents in their hollow inner cavities and release them at a later stage in a controlled manner. Even though several different routes, such as layer-by-layer deposition of polyelectrolytes onto a template core, ${ }^{4}$ microemulsion polymerization, ${ }^{5}$ and self-assembly of block copolymers in a selective solvent, ${ }^{6}$ have been successfully developed, these strategies require the templates to be removed to create a hollow interior or need greater quantities of surfactants to form nanosized micelles. Furthermore, most of the hollow nanocapsules described to date are ill-suited for biomedical application, and the lack of biocompatibility and degradability is of another important concern. Therefore, the use of natural polymer with properties desirable for many practical considerations in the field of biomedicals is of most interest.

As was well recognized, chitosan (CS), $\beta$-(1,4)-link glucosamine unit, is produced by deacetylation of chitin, which is extracted from the shells of crabs, shrimp, and krill. It exhibits several characteristics such as biocompatibility, nontoxicity, and bioadhesivity which make it as an ideal material for biomedical uses, such as drug delivery. ${ }^{7}$ However, its poor solubility in

\footnotetext{
* Corresponding author: Tel +886-3-5712121(ext. 55391); E-mail: deanmo_liu@yahoo.ca.

National Chiao Tung University.

* National Yang-Ming University.
}

water and common organic solvents has so far limited its widespread utilization. The reactive amino groups in the backbone of chitosan make it possible to chemically conjugate with various biological molecules. ${ }^{8}$ As a result, there have been many works about the methods to enhance the solubility of chitosan, one of which was derivatization. For example, the introduction of carboxyl groups to chitosan derivatives led to the anionic or amphotheric properties, are water-soluble, and are attracting much attention as metal collecting materials and biomaterials. ${ }^{9}$ Hence, hydrophilically, ${ }^{10}$ hydrophobically, ${ }^{11}$ and amphiphilically ${ }^{12}$ modified chitosan derivatives are being studied to form the monodisperse self-aggregated nanoparticles by sonication in aqueous media due to noncovalent association arising from intra- or/and intermolecular interactions among hydrophobic segments in aqueous media. However, in these studies, the formation of hollow structure is more difficult than that of particle structure.

In this study, a new type of chitosan hollow structure, i.e., carboxymethyl-hexanoyl chitosan (CHC), which was modified first by hydrophilic carboxymethylation to increase the flexibility of chitosan molecular chains in water ${ }^{13}$ followed by hydrophobic modification with hexanoyl groups to add amphiphilic character, was employed to study its self-aggregation behavior to form nanocapsule in aqueous solution and nanostructural evolution. The stability of nancapsules and formation mechanism of the $\mathrm{CHC}$ macromolecules were explored through the use of critical aggregation concentration (cac), zeta potential, electron microscopy, and dynamic light scatter (DLS). By taking the advantage of self-aggregation nature, the $\mathrm{CHC}$ was employed to encapsulate doxorubicin (DOX), an anticancer agent of broad spectrum with reasonable therapeutic index and intriguing biological and physicochemical actions, ${ }^{14}$ to further understand its loading efficiency and release behavior.

\section{Experimental Section}

2.1. Materials. Chitosan $\left(M_{\mathrm{w}}=215000 \mathrm{~g} / \mathrm{mol}\right.$, deacetylation degree $=85-90 \%$ ) was supplied from Aldrich-Sigma. 2-Propanol, sodium hydroxide, chloroacetic acid, and hexanoyl anhydride were 
Table 1. Sample Name and Corresponding Estimation of Substitution Degree by ${ }^{1} \mathbf{H} \mathbf{N M R}^{15}$

\begin{tabular}{lcc}
\hline & \multicolumn{2}{c}{ degree of substitution } \\
\cline { 2 - 3 } & carboxymethyl group & hexanoyl group \\
\hline CC & 0.5 & 0 \\
CHC-1 & 0.5 & 0.13 \\
CHC-2 & 0.5 & 0.26 \\
CHC-3 & 0.5 & 0.33 \\
CHC-4 & 0.5 & 0.48
\end{tabular}

purchased from Sigma Co. All other chemical reagents in the study were analytical grade.

2.2. Synthesis of CHC. According to our previous report, ${ }^{15} \mathrm{CHC}$ can be successfully prepared through following steps. Briefly, $5 \mathrm{~g}$ of chitosan was suspended in 2-propanol $(50 \mathrm{~mL})$ at room temperature while being stirred for $30 \mathrm{~min}$. The resulting suspension was gently mixed with $12.5 \mathrm{~mL}$ of $\mathrm{NaOH}$ solution. The mixture containing $\mathrm{NaOH}$ of $13.3 \mathrm{M}$ was mixed with $25 \mathrm{~g}$ of chloroacetic acid to prepare carboxymethyl chitosan (CC) sample with a high degree of carboxymethyl substitution. The obtained and dried sample $(2 \mathrm{~g})$ was dissolved in distilled water $(50 \mathrm{~mL})$ while being stirred for $24 \mathrm{~h}$. These resulting solutions were mixed with methanol $(50 \mathrm{~mL})$, followed by the addition of hexanoyl anhydride at concentration of $0.2,0.3,0.4$, and $0.5 \mathrm{M}$ for the $\mathrm{CHC}$ samples with different degrees of hexanoyl substitution. After the reaction time of $12 \mathrm{~h}$, the resulting solutions were collected by dialysis membrane (Sigma Chemical Co.) after dialysis with ethanol solution $(25 \%$ $\mathrm{v} / \mathrm{v}$ ) for $24 \mathrm{~h}$. CHC samples with various degrees of hexanoyl substitution $(\mathrm{DH})$ were named and are shown in Table 1 . The sites and degrees of substitution were confirmed by ${ }^{1} \mathrm{H}$ NMR analysis in pervious work. ${ }^{15}$

2.3. Preparation of CHC Hollow Nanocapsules. A $100 \mathrm{mg} /$ $\mathrm{mL}$ CHC sample was suspended in distilled water under gentle shaking at $25^{\circ} \mathrm{C}$ for $24 \mathrm{~h}$, followed by ultrasonication using a probe type sonifier (Automatic Ultrasonic Processor UH-500A, China) at $30 \mathrm{~W}$ for $2 \mathrm{~min}$. The sonication was repeated three times to get an optically clear solution. To inhibit the heat buildup during sonication, the pulse function was used (pulse on $5.0 \mathrm{~s}$; pulse off $1.0 \mathrm{~s})$.

2.4. Characterization. Zeta potential measurements of the nanocapsules were determined with a Malvern Zreasizer 169 HS3000 photon correlation spectrometer with an applied 170 voltage of $100 \mathrm{~V}$. The mean size and size distribution of the nanocapsules were measured by dynamic light scattering (DLS) nanoparticle size analyzer (LB-550, HORIBA, Japan). All measurements were done with a wavelength of $633.0 \mathrm{~nm}$ at $25^{\circ} \mathrm{C}$ with an angle detection of $90^{\circ}$. Each sample was repeatedly measured three times. Morphological evaluation of the hollow nanocapusles was performed by transmission electron microscopy (TEM) (JEOL2100, Japan) and scanning electron microscopy (SEM) (S6500, JEOL, Japan). Sample solutions were dropped onto the carbon-coated 300 mesh copper grids and dried at $50{ }^{\circ} \mathrm{C}$ and then examined without being stained for TEM analysis. For SEM observation, sample was suspended into anhydrous ethanol and then dip-coated on the silicon substrate. After evaporation at $50{ }^{\circ} \mathrm{C}$ for $24 \mathrm{~h}$, dried samples were coated with gold ( $\sim 20 \mathrm{~nm}$ thickness) for analysis.

2.5. Self-Aggregation Behavior of CHC. The pyrene solution $\left(1.0 \times 10^{-4} \mathrm{M}\right)$ in methanol was added into the test tubes and evaporated under a stream of nitrogen gas to remove the solvents. Then, solutions of CHC self-aggregates in distilled water were added into the above test tubes, bringing the final concentration of pyrene to $1.0 \times 10^{-6} \mathrm{M}$, which was nearly equal to the solubility of pyrene in water at $22{ }^{\circ} \mathrm{C}$. ${ }^{16}$ The mixtures were sonicated for 30 min in an ultrasonic bath and shaken in a shaking air bath for $1 \mathrm{~h}$ at room temperature. Pyrene emission spectra were obtained using a fluorescence spectrophotometer (Hitachi FL-4500, Japan). The probe was excited at $343 \mathrm{~nm}$, and the emission spectra were recorded in the range of $350-500 \mathrm{~nm}$ at an integration time of $1.0 \mathrm{~s}$. The excitation and emission slit opening were 10 and 2.5 $\mathrm{nm}$, respectively.

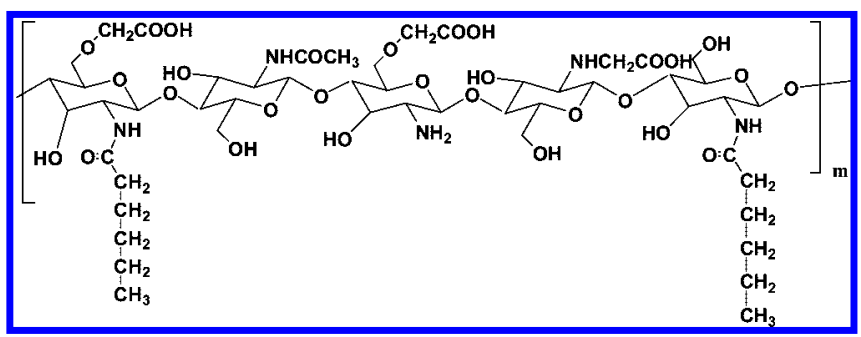

Figure 1. Molecular structure of carboxymethyl-hexanoyl chitosan (CHC).

2.6. Drug Loading Efficiency. Drug-loaded hollow nanocapsules were prepared by dissolving $20 \mu \mathrm{g} / \mathrm{mL}$ of DOX in $20 \mathrm{~mL}$ acquired nanocapsule suspension before ultrasonication. Insoluble, free DOX was removed by centrifugation at $2000 \mathrm{rpm}$ and $4{ }^{\circ} \mathrm{C}$ for $5 \mathrm{~min}$. The drug-containing nanocapsules were then separated from the aqueous solution by centrifugation at $15000 \mathrm{rpm}$ and $4{ }^{\circ} \mathrm{C}$ for 15 min. Drug concentration in the supernatant was analyzed by ultraviolet absorption (UV) at wavelength of $490 \mathrm{~nm}$, a strong absorption band of DOX, with reference to a calibration curve on a UV-vis spectrometer (SP-8001, Metertech Inc.). The measurements were performed in triplicate. The amount of the drug in the hollow nanocapsules could be calculated by the total amount of DOX subtracting the residual DOX in the supernatant. The encapsulation efficiency (EE) was obtained as follows:

$$
\mathrm{EE}=(A-B) / C \times 100
$$

where $A$ is the total amount of the DOX, $B$ is the amount of DOX remaining in the supernatant, and $C$ is the weight of the nanocapsules.

2.7. DOX Release. A solution of DOX or DOX-loaded nanocapsules ( $\sim 20 \mu \mathrm{g} / \mathrm{mL}$ DOX equiv) was added to a Slid-A-Lyzer dialysis cassette (Pierce) with a molecular weight cutoff of 10000. The solution was dialyzed at $37{ }^{\circ} \mathrm{C}$ against $0.1 \mathrm{M} 100 \mathrm{~mL}$ buffer solution. The solution was removed periodically from the dialysis cassette and characterized using an absorbance at a wavelength of $490 \mathrm{~nm}$ to determine the amount of DOX in the solution, and then the solution was poured back to the dialysis cassette.

\section{Results and Discussion}

3.1. Formation of CHC Nanoaggregates. The molecular structure of $\mathrm{CHC}$ is given in Figure 1, and CHC samples with various degrees of hexanoyl substitution $(\mathrm{DH})$ were confirmed by ${ }^{1} \mathrm{H}$ NMR analysis ${ }^{15}$ as given in Table 1 . After dispersing in water, the $\mathrm{CHC}$ conjugates formed self-assembled nanometric aggregates by ultrasonication. The fluorescence probe technique was used to investigate the self-aggregation behavior of the $\mathrm{CHC}$ at a molecular level, and pyrene was chosen as a fluorescence probe. Pyrene shows only small fluorescence intensity in a polar environment (such as water) due to its poor solubility and selfquenching, but strongly emits when the hydrophobic microdomains are formed in an aqueous solution because the pyrene molecules show high affinity to these hydrophobic microdomains. Therefore, pyrene is often used as a fluorescence probe to monitor the self-aggregation behavior of surfactants and/or polymers. ${ }^{17}$ In addition, the intensity ratio of the first peak ( 372 $\mathrm{nm})$ and the third peak $(385 \mathrm{~nm}), I_{372} / I_{385}$, in its fluorescent spectrum is quite sensitive to the environment surrounding the pyrene molecules and has been frequently used as an indicator for a subtle change of its environment. Hence, the critical aggregation concentration (cac), which is defined as the threshold concentration of self-aggregate formation by intraand /or intermolecular association, can be determined from the change of the $I_{372} / I_{385}$ value of pyrene in the presence of polymeric amphiphiles. Figure 2 illustrates the changes of the $I_{372} / I_{385}$ values as a function of the concentrations of $\mathrm{CHC}-4$ and CC. For CHC-4, the $I_{372} / I_{385}$ values were close to the value 


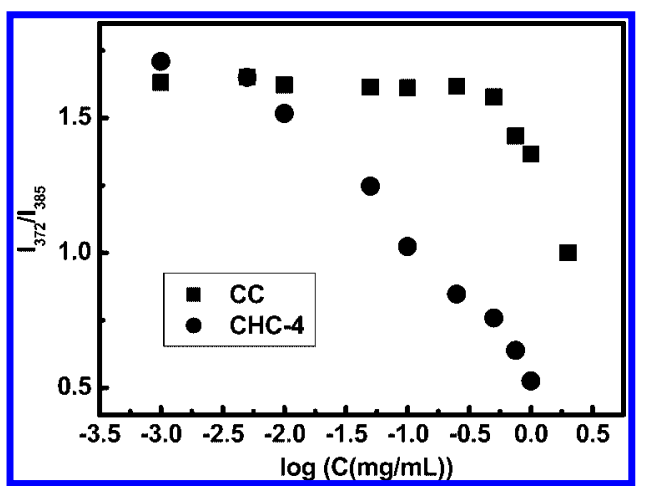

Figure 2. Change of the intensity ratio $\left(I_{372} / I_{385}\right)$ from excitation spectra of pyrene $\left(1.0 \times 10^{-6} \mathrm{~mol} / \mathrm{L}\right)$ with various concentrations of $\mathrm{CC}$ and CHC-4.

Table 2. Characterization of $\mathrm{CC}$ and $\mathrm{CHC}$ Self-Assembled Hollow Nanocapsules

\begin{tabular}{lcccc}
\hline samples & $\begin{array}{c}\mathrm{cac}^{a} \times 10^{-2} \\
(\mathrm{mg} / \mathrm{mL})\end{array}$ & $\begin{array}{c}\text { zeta potential }^{b} \\
(\mathrm{mV})\end{array}$ & $\begin{array}{c}\text { mean size } 1^{c} \\
(\mathrm{~nm})\end{array}$ & $\begin{array}{c}\text { mean size } 2^{c} \\
(\mathrm{~nm})\end{array}$ \\
\hline CC & 25.0 & $-42.8 \pm 4.26$ & $19.8 \pm 1.2$ & $189.2 \pm 2.4$ \\
CHC-1 & 9.21 & $-38.4 \pm 2.27$ & $21.5 \pm 1.6$ & $210.5 \pm 1.9$ \\
CHC-2 & 3.17 & $-27.5 \pm 3.49$ & $22.3 \pm 0.7$ & $209.3 \pm 1.4$ \\
CHC-3 & 0.91 & $-24.4 \pm 0.48$ & $22.9 \pm 1.1$ & $210.9 \pm 0.7$ \\
CHC-4 & 0.40 & $-23.1 \pm 0.86$ & $23.5 \pm 0.7$ & $222.7 \pm 2.0$
\end{tabular}

${ }^{a}$ Critical aggregation concentration determined from $I_{372} / I_{385}$ data. ${ }^{b}$ The zeta potential (mean value \pm standard deviation) measured by an photon correlation spectrometer with three times. ${ }^{c}$ The size and size distribution (mean value \pm standard deviation) determined by the dynamic light scattering with three times.

(1.72) of pyrene in water at low concentrations ${ }^{18}$ and then followed by a linear decrease with further increasing concentration. The cac can then be determined by the intercept of two straight lines. ${ }^{19}$ It was found that the CHC-4 composition demonstrated the lowest cac, i.e., $0.004 \mathrm{mg} / \mathrm{mL}$, among the others and is lower than the critical micelle concentration of many low-molecular-weight surfactants reported, e.g., sodium dodecyl sulfate. ${ }^{20}$ Accordingly, this finding suggests that the $\mathrm{CHC}$ nanoaggregates prepared in this work should be thermodynamically stable in aqueous solution, and this has been further evidenced by the fact that those nanocapsules exhibited excellent colloidal stability in PBS over a long-term storage for at least 1 month in refrigerator.

However, for CC, the $I_{372} / I_{385}$ values decreased slightly only at high concentration $(0.25 \mathrm{mg} / \mathrm{mL})$, indicating difficulty for the CC molecules to aggregate in the studied range of concentration. Moreover, since from Table 2 the cac values of the $\mathrm{CHC}$ nanoaggregates were decreased with the increase of $\mathrm{DH}$; it can thus be deduced from the observation of the self-aggregate ability between $\mathrm{CC}$ and $\mathrm{CHC}$ that the aggregation of $\mathrm{CHC}$ in water was due to the incorporation of hydrophobic groups by increasing the degree of hexanoyl substitution. It was known that the increase of hydrophobicity in amphiphatic polymer causes the difficulty to dissolve in aqueous solution because the energy is essential to cut the H-bonding in water molecules (need to do work). Therefore, the dissolution of hydrophobic groups in water would result in the increase of the surface free energy. In order to decrease the energy, the hydrophobic groups trend to aggregate. Hence, $\mathrm{CHC}-4$, having the highest $\mathrm{DH}$, is able to form nanoaggregates at a very low concentration (e.g., $0.004 \mathrm{mg} / \mathrm{mL}$ ) because increase of the resulting surface energy is the greatest. On the contrary, for $\mathrm{CC}$ with the least hydrophobicity, the required concentration to form nanoaggregates is much higher (e.g., $0.25 \mathrm{mg} / \mathrm{mL}$ ) than that of the CHC. This appears to indicate that the self-aggregate ability can be effectively enhanced with increasing hydrophobic nature of the modified chitosan in an aqueous environment.

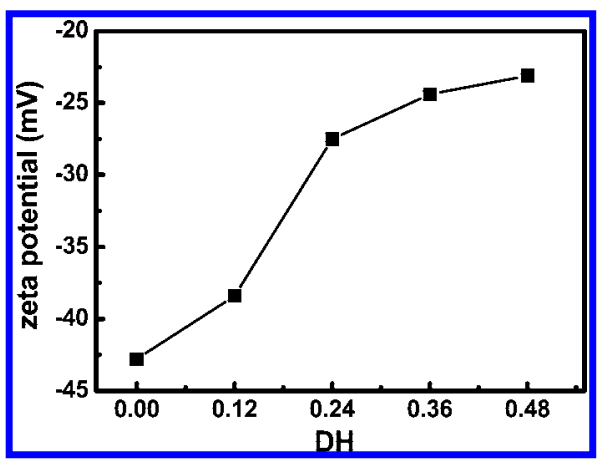

Figure 3. Zeta potential of carboxymethyl-hexanoyl chitosan (CHC) with different DH.

The zeta potentials of the resulting $\mathrm{CHC}$ self-aggregated nanoparticles in water were determined and showed to be negatively charged (Table 2), suggesting that the negatively charged carboxymethyl groups in the $\mathrm{CHC}$ molecules were mainly distributed on the outer surface of the nanoparticles contributing to the hydrophilic potential. The absolute value of the zeta potential of the $\mathrm{CHC}$ nanoparticles decreased from 42.8 to $23.1 \mathrm{mV}$ with $\mathrm{DH}$ increasing from 0 to 0.48 , as shown in Figure 3. The change of the zeta potential (or surface charge) of the $\mathrm{CHC}$ nanoparticles is rapid while the substitution is below 0.26; however, it turns out to reach a plateau stage with further increase in the DH level over 0.33, suggesting the addition of the hexanoyl group may reach a level of saturation. The sharp decrease of the zeta potential from -38.4 to $-27.5 \mathrm{mV}$ when the $\mathrm{DH}$ is increasing from 0.13 to 0.26 suggests a reduction, albeit not significant, of the electrostatic repulsion, which, as a consequence, may cause gradual agglomeration of the $\mathrm{CHC}$ molecules to form larger particles while dispersing in water.

Figure 4 shows the transmission electron microscopy (TEM) images of the $\mathrm{CHC}$ nanoaggregates. The nanoaggregates show a perfect spherical geometry and have a size ranging from 20 to100 $\mathrm{nm}$ with increase of DH. In contrast, the self-assembled CC nanoaggregates (Figure 4a) show a relatively uniform size distribution of around $20 \mathrm{~nm}$ in diameter. While increasing the $\mathrm{DH}$ to 0.48 (CHC-4), it was found that the size of nanoaggregate is increased to around $100 \mathrm{~nm}$, as depicted in Figure $4 \mathrm{~b}$. In addition, the mean size of the $\mathrm{CHC}$ nanoaggregates measured by dynamic light scattering (DLS) exhibits two size groups, as listed in Table 2: one corresponds to smaller particle size of around $40 \mathrm{~nm}$ (i.e., mean size 1), and the other is larger particle size of around $200 \mathrm{~nm}$ (mean size 2). The smaller particle size may be caused by the coil of the CHC single chain in the solution. ${ }^{21}$ However, with increase of $\mathrm{DH}$, the enhanced hydrophobic interaction and, in the meantime, reduced intermolecular repulsive force both facilitate self-aggregation, thus increasing the size of the aggregates. Such a bimodal size distribution of the $\mathrm{CHC}$ nanoparticle suggests that the nanoaggregates formed simultaneously by the coil of the $\mathrm{CC}$ single chain and/or by the hydrophobic interaction among $\mathrm{CHC}$ macromolecules in the solution. It was obtained from Figure 5 that the CC shows a high fraction $(>90 \%)$ of the nanoaggregates having a size of $40 \mathrm{~nm}$, suggesting that it is difficult to form larger aggregate with lower degree of hydrophobic substitution (i.e., $\mathrm{DH}<0.13$ ). However, as $\mathrm{DH}$ increased up to 0.26 , the nanoaggregates show a high fraction of mean size 2 (around $200 \mathrm{~nm}$ ), indicating that a critical concentration of hexanoyl substitution in the range between 0.13 and 0.26 dominates the self-assemble behavior of the CHC macromolecules.

3.2. Nanostructural Development of the CHC Nanoaggregates. In Figure 6a, the hexanoyl-modified carboxymethyl chitosan has the amphiphilic property with hydrophile and hydrophobe. On the basis of the polycore model proposed by 


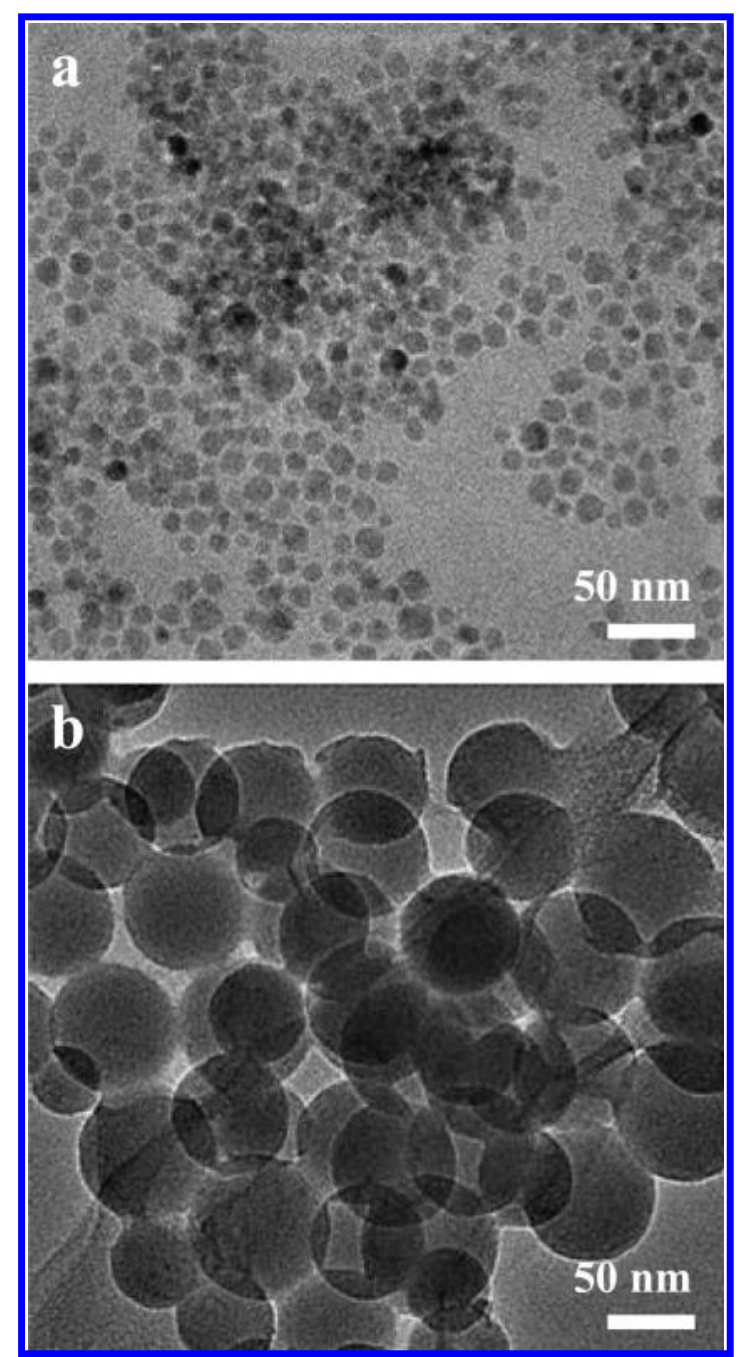

Figure 4. TEM images of self-aggregates prepared from (a) CC and (b) CHC-4 by ultrasonication in water.

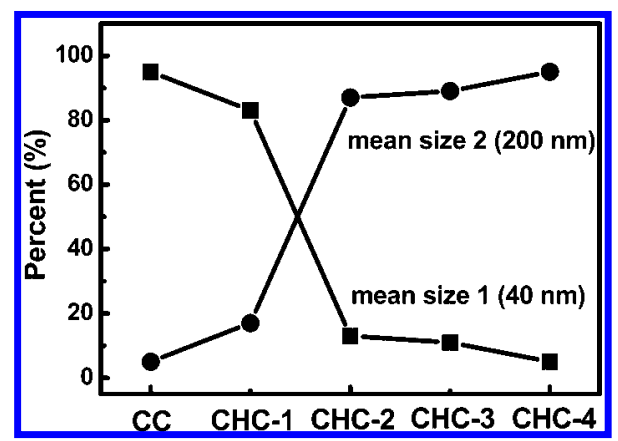

Figure 5. Fraction of mean size 1 and 2 of $\mathrm{CC}$ and $\mathrm{CHC}$ nanoaggregates.

Wang et al., ${ }^{22}$ for hydrophobically modified polysaccharides, the hydrophobic microdomains are formed by the association of hydrophobic group, and the polysaccharide backbones coil to form the hydrophilic shells outside these hydrophobic microdomains; thus, a minimal energy state is attained in aqueous media. For $\mathrm{CC}$ and $\mathrm{CHC}-1$, the hydrophobic interaction provides the driving force for self-aggregation as supported to be a combination of the effects of intermolecular H-bonding of $\mathrm{CC}$ (e.g., the presence of carboxylic, hydroxyl, and amino groups on the CC chains); electrostatic repulsions between $\mathrm{COO}-$ groups on the $\mathrm{CC}$ chains and hydrophobic interaction among the hydrophobic moieties in $\mathrm{CC}$ such as acetyl groups and glucosidic rings. ${ }^{10}$ Hence, for the $\mathrm{CHC}$ with lower $\mathrm{DH}$, the

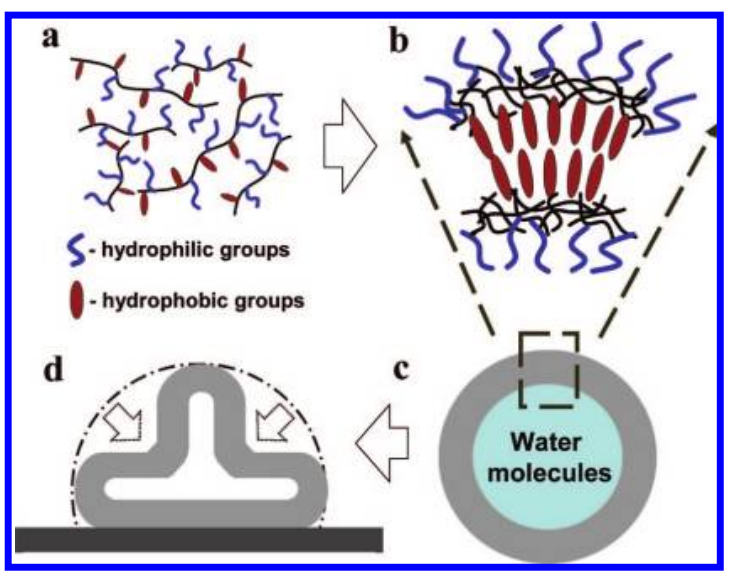

Figure 6. Schematic illustration of formation process of $\mathrm{CHC}$ hollow nanocapsules.

particle-like aggregated structure of $\mathrm{CHC}$ was formed. However, from a recent investigation using hydrophobic acyl group of various carbon lengths, we found that a structural change from particle to hollow capsule with increasing the $\mathrm{DH}$ of $\mathrm{CHC}$ was strongly affected by the variation of the physical state of water molecules within the aggregates (submitted in press). Accordingly, the increase of bound water within $\mathrm{CHC}$ matrix with increasing DH enhances difficulty in the formation of hydrophobic core and becomes structurally unstable. In contrast, with increase of free energy caused by increasing hydrophobicity, the $\mathrm{CHC}$ tends to self-aggregate to form a bilayer structure with hydrophilic-hydrophobic-hydrophilic configuration, as schematically illustrated in Figure $6 \mathrm{~b} .^{23}$ The amphiphilic potential of the $\mathrm{CHC}$ with higher $\mathrm{DH}$ is then thermodynamically favorably to drive the resulting $\mathrm{CHC}$ macromolecules to selfassemble into a bilayer structure with water molecules encapsulated inside the core phase while both interior and exterior regions of the shell are stabilized by the hydrophilic groups, forming a spherical architecture with minimal surface energy, as schematically shown in Figure 6c.

However, an increase in entropy change due to the formation of nanoaggregate in aqueous environment has to be compromised thermodynamically. Although there are little evidence or supports that are available in the literature in similar materials systems, an alternative mechanism proposed by Moroi $^{24}$ appears feasible. Moroi involved the peculiar properties of water by recognizing that the water molecules in the liquid state have a structure of hydrogen bonds similar to that of ice; the cavities in the structure are large enough to accommodate a hydrophobic group, such as a hydrocarbon chain. Thus, occupation of a cavity by hydrophobic solute hinders the movement of free water molecules, which therefore remain stationary for longer periods, i.e., improved stability of the colloidal system. In order words, the water molecules surrounding a hydrophobic solute become more ordered than bulk water molecules and have lower entropy. Accordingly, the water molecules become more ordered around the hydrophobic solute with an increase in hydrophobicity in order to accommodate the variation of system entropy while the nanoaggregates were underdeveloping.

Though the morphology of the nanoaggregates show spherical shape from TEM results, the scanning electron microscopy (SEM) images show entirely different morphologies of the nanoaggregates, as shown in Figure 7, where the nanoaggregates displayed collapsed structures. This variation is mainly caused by the difference of the preparation methods. For TEM sample preparation, the $\mathrm{CHC}$ aggregates are subjected to ambient drying, where the excellent water absorption and water retention capability, especially the crystal water binding with the molecular structure keep the $\mathrm{CHC}$ nanoaggregate its structural 


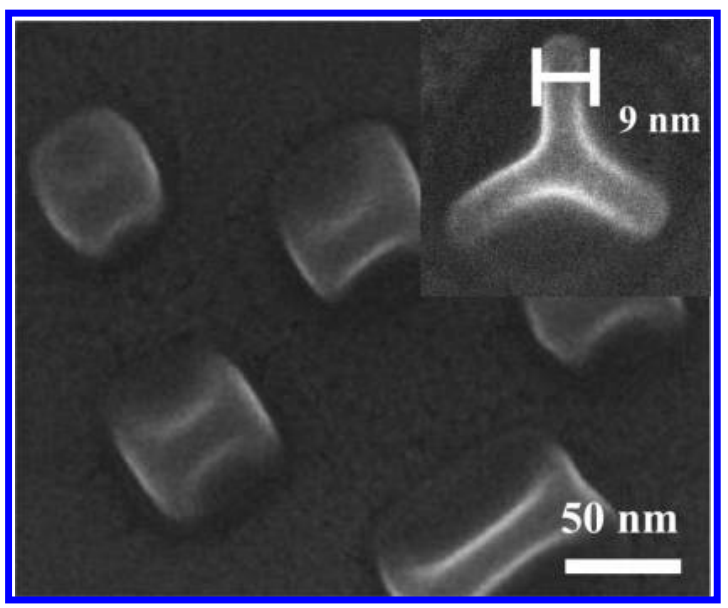

Figure 7. SEM images of CHC-4 nanocapsules.

integrity. However, when the water, i.e., both free water (i.e., unbound water) and crystal water (i.e., bound water) being removed from the $\mathrm{CHC}$ macromolcules using ethanol through extensive dehydration, structural integrity of the nanoaggregates can no longer preserve. It collapsed as a flatten basketball. This morphological change suggests a hollow structure evolved upon the first formation of the CHC nanoaggregates (Figure 6d), indicating a capsule-like nanostructure, where a core-shell configuration developed. From the collapsed morphology, the thickness of the shell layer of the collapsed nanocapsules, from the image inserted in Figure 7 (a combination of two shells of the nanocapsule) is about $9 \mathrm{~nm}$; the layer thickness of the single shell is ca. to be $4.5 \mathrm{~nm}$. Such a small scale of the shell thickness may explain the reason that the core-shell structure of the $\mathrm{CHC}$ nanocapsules is hard to distinctly differentiate from TEM analysis under the resolution aforementioned.

3.3. DOX Loading and Release Behavior of CHC Nanocapsules. To investigate the drug-loading capacity of the $\mathrm{CHC}$ nanocapsules, the model drug, doxorubicin (DOX), was loaded into the $\mathrm{CHC}$ nanocapsules. Figure 8 a shows the encapsulation efficiency (EE) of DOX from CHC nanocapsules with different DH. As reported by Kitaeva et al., ${ }^{25}$ since DOX molecule contains an amino group with a $\mathrm{p} K_{\mathrm{a}}$ of 8.6 , it is expected that DOX can form polyelectrolyte complex with the carboxymethyl groups of $\mathrm{CC}$ and $\mathrm{CHC}$. Hence, for the $\mathrm{CC}$ (where its DH is zero), it shows a lower encapsulation efficiency of around $26.3 \%$. However, as increase the level of hydrophobic substitution, the DOX encapsulation efficiency increased from 26.3 to $46.8 \%$ as the $\mathrm{DH}$ increased form 0 to 0.48 . The improved encapsulation efficiency with increase of $\mathrm{DH}$ of the $\mathrm{CHC}$ should be caused by the larger reservoir space and the increase of the hydrophobic interaction of inner shell, which restricts the outward diffusion of DOX from nanocapsules.

The release of DOX from the $\mathrm{CHC}$ nanocpasules was investigated using a dialysis procedure. A solution of the DOXloaded $\mathrm{CHC}$ nanocapsules in a dialysis cassette was dialyzed against $100 \mathrm{mM}$ buffer, and the solution in the cassette was sampled at various times over a 7 day period to determine the amount of DOX remaining in the nanocapsules. Figure $8 \mathrm{~b}$ shows the release profiles of DOX and DOX-loaded CHC nanocapsules with different levels of DH. It was observed that DOX and DOX-loaded CC had a faster diffusion rate with essentially complete release after $24-36 \mathrm{~h}$. This is consistent with the expected rate of diffusion for low molecular weight molecules across the dialysis membrane. However, DOX-loaded CHC nanocapsules show a two-step release profile. In the first step $(0-12 \mathrm{~h})$, the rapid release of DOX may caused by the diffusion of DOX on the surface of nanocapsules out of the dialysis

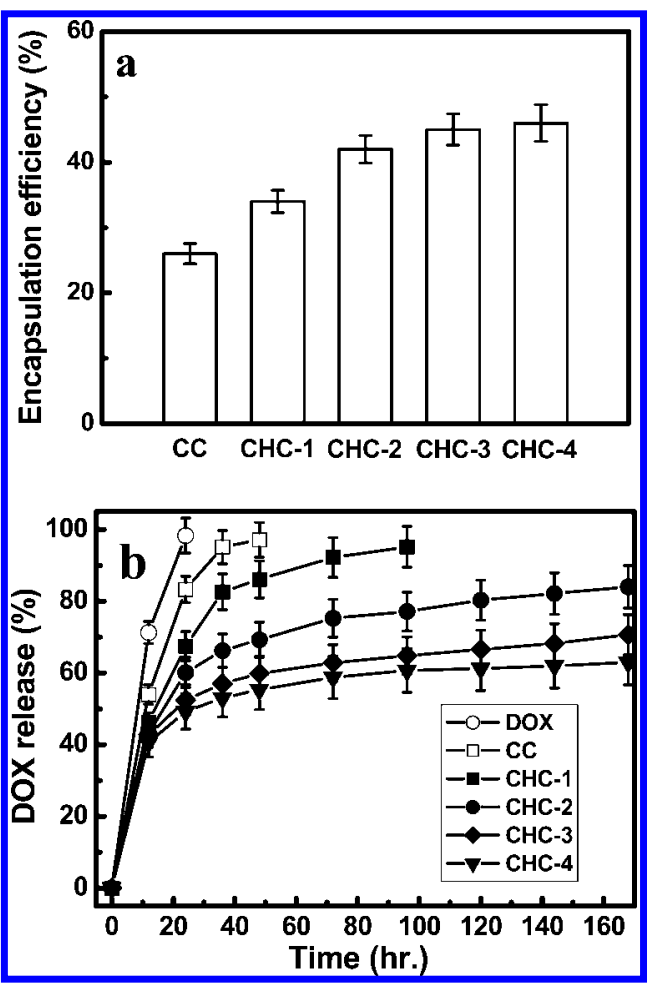

Figure 8. (a) DOX encapsulation efficiency and (b) DOX release profiles of CHC hollow nanocapsules.

membrane. Then, the followed a relatively slow release, which may be dominated by the $\mathrm{DH}$ of $\mathrm{CHC}$ nanocapsules. It can be found that CHC-1 showed a total release period of about $96 \mathrm{~h}$. By contrast, with the increase of DH (i.e., CHC-2, CHC-3, and CHC-4), the entrapped DOX showed a continuous release over 7 days. It is reasonably believed that the increase of hydrophobic groups enhances encapsulation efficiency and payload of the DOX within $\mathrm{CHC}$ nanocapsules. These results indicate that these CHC self-assembled hollow nanocapsules can be a suitable candidate for controlled DOX delivery for anticancer purposes.

\section{Conclusion}

In present study, we demonstrated a simple and direct method that amphiphilic CHC hollow nanocapsules were developed in aqueous system without the aid of surfactants, organic solvents, emulsion phases, or template cores. In addition, the particle size, surface charge, and critical aggregation concentration (cac) of the CHC self-assembled hollow nanocapsules with different degree of hydrophobic substitution were systematically investigated. The results of SEM and TEM proved that the selfassembled CHC nanocapsules exhibited spherical morphology and a core-shell configuration. The formation of $\mathrm{CHC}$ selfassembled hollow nanocapsules is due to the hydrophobic interactions of hexanoyl groups in aqueous solution, and the negatively charged carboxymethyl groups also play an important role in the morphology and the stability of nanocapsules. The loading of doxorubicin drug can be improved by increase of $\mathrm{DH}$ for a sustained release over a reasonable period of time.

Acknowledgment. This work was financially supported by the National Science Council of the Republic of China, Taiwan, under Contracts NSC96-2627-B-009-006 and NSC-96-2113-M-009-027MY2.

\section{References and Notes}

(1) Panyam, J.; Labhasetwar, V. Adv. Drug Delivery Rev. 2003, 55, 32947. 
(2) Hu, Y.; Zhang, L. Y.; Cao, Y.; Jiang, X. Q. Biomacromolecules 2004, 5, 1756-1762.

(3) Zheng, R.; Liu, G. Macromolecules 2007, 40, 5116-5121.

(4) Caruso, F.; Caruso, R. A.; Mohwald, H. Science 1998, 282, 11111114.

(5) Hentze, H.-P.; Kaler, E. W. Curr. Opin. Colloid Interface Sci. 2003, $8,164$.

(6) Huang, H.; Remsen, E. E.; Kowalewski, K. L.; Wooley, K. L. J. Am. Chem. Soc. 1999, 121, 3805.

(7) Fini, A.; Orienti, I. Am. J. Drug Delivery 2003, 1, 43-59.

(8) Lee, M.; Nah, J. W.; Kwon, Y.; Koh, J. J.; Lo, K. S.; Kim, S. W. Pharm. Res. 2001, 18, 427-431.

(9) Muzzarelli, R. A. A.; Muzzarelli, C. Adv. Polvm. Sci. 2005, 186, 151209.

(10) Zhu, A.; Chan-Park, M. B.; Dai, S.; Li, L. Colloids Surf., B 2005, 43 143-149.

(11) Chae, S. Y.; Son, S.; Lee, M.; Jang, M.-K.; Nah, J.-W. J. Controlled Release 2005, 109, 330-344.

(12) Suia, W.; Songa, G.; Chenb, G.; Xuc, G. Colloids Surf., A 2005, 256, $29-33$.

(13) Zhao, A. J.; Yuan, X. B.; Chang, J. Polym. Bull. 2004, 4, 59-63.
(14) Young, C.; Ozols, R. F.; Myers, C. E. New Engl. J. Med. 1981, 305, 139-153.

(15) Liu, T. Y.; Chen, S. Y.; Lin, Y. L.; Liu, D. M. Langmuir 2006, 22, 9740-9745.

(16) Wilhelm, M.; Zhao, C.; Wang, Y.; Xu, R.; Winnik, M. A.; Mura, J. Macromolecules 1991, 24, 1033-1044.

(17) Amiji, M. M. Carbohvdr. Polvm. 1995, 26, 21-213.

(18) Dong, D. C.; Winnik, M. A. Can. J. Chem. 1984, 62, 2560-2565.

(19) Lee, K. Y.; Jo, W. H. Langmuir 1998, 14, 2329-2332.

(20) Rahman, A.; Brown, C. W. J. Appli. Polym. Sci. 2003, 28, 13311334

(21) Peng, X.; Zhang, L. J. Am. Chem. Soc. 2007, 23, 10493-10498.

(22) Wang, Y.; Liu, L.; Weng, J.; Zhang, Q. Carbohvdr. Polvm. 2007, 69, 597-606.

(23) Li, G.; Zhuang, Y.; Mu, Q.; Wang, M.; Fang, Y. Carbohvdr. Polvm. 2008, 72, 60-66.

(24) Moroi, Y. Micelles Theoretical and Applied Aspects; Plenum Press: New York, 1992.

(25) Kitaeva, M. V.; Melik-Nubarov, N. S.; Menger, F. M. Langmuir 2004, 20, 6575-6579.

MA8002399 